\title{
Enhancer methylation dynamics contribute to cancer plasticity and patient mortality
}

\author{
Rachel E. Bell,, ${ }^{1,4}$ Tamar Golan, ${ }^{1,4}$ Danna Sheinboim, ${ }^{1}$ Hagar Malcov, ${ }^{1}$ David Amar, ${ }^{2}$ \\ Avi Salamon, ${ }^{3}$ Tamar Liron, ${ }^{1}$ Sahar Gelfman, ${ }^{1,5}$ Yankel Gabet, ${ }^{3}$ Ron Shamir, ${ }^{2}$ \\ and Carmit Levy ${ }^{1}$ \\ ${ }^{7}$ Department of Human Genetics and Biochemistry, Sackler Faculty of Medicine, Tel Aviv University, Tel Aviv 69978, Israel; ${ }^{2}$ Blavatnik \\ School of Computer Science, Tel Aviv University, Tel Aviv 69978, Israel; ${ }^{3}$ Department of Anatomy and Anthropology, Sackler Faculty of \\ Medicine, Tel Aviv University, Tel Aviv 69978, Israel
}

\begin{abstract}
During development, enhancers play pivotal roles in regulating gene expression programs; however, their involvement in cancer progression has not been fully characterized. We performed an integrative analysis of DNA methylation, RNA-seq, and small RNA-seq profiles from thousands of patients, including 25 diverse primary malignances and seven body sites of metastatic melanoma. We found that enhancers are consistently the most differentially methylated regions (DMR) as cancer progresses from normal to primary tumors and then to metastases, compared to other genomic features. Remarkably, identification of enhancer DMRs (eDMRs) enabled classification of primary tumors according to physiological organ systems, and in metastasis eDMRs are the most correlated with patient outcome. To further understand the eDMR role in cancer progression, we developed a model to predict genes and microRNAs that are regulated by enhancer and not promotor methylation, which shows high accuracy with chromatin architecture methods and was experimentally validated. Interestingly, among all metastatic melanoma eDMRs, the most correlated with patient survival were eDMRs that "switched" their methylation patterns back and forth between normal, primary, and metastases and target cancer drivers, e.g., KIT. We further demonstrated that eDMR target genes were modulated in melanoma by the bone metastasis microenvironment, suggesting that eDMRs respond to microenvironmental cues in metastatic niches. Our findings that aberrant methylation in cancer cells mostly affects enhancers, which contribute to tumor progression and cancer cell plasticity, will facilitate development of epigenetic anticancer approaches.
\end{abstract}

[Supplemental material is available for this article.]

The landscape of DNA methylation undergoes global changes in many cancers (Jones 2012) which contribute to genomic instability (Pogribny and Beland 2009), facilitate genetic mutations (You and Jones 2012), and alter gene expression programs (Easwaran et al. 2014). Most studies of DNA methylation changes in cancers have focused on promoter regions, since hypermethylation of promoters is a key mechanism for gene silencing (Esteller 2007). Less attention has been given to aberrant DNA methylation in other regions of the genome, such as enhancers (Aran and Hellman 2013; Marzese et al. 2014; Ziller et al. 2013; Brocks et al. 2014), and to its influence on gene expression in cancer.

During development, enhancers play pivotal roles in regulating expression programs (Hnisz et al. 2013) and are characterized by tissue-specific chromatin marks (Heintzman et al. 2009), and their activation corresponds with nucleosome and methylation loss (Zhou et al. 2011; Buecker and Wysocka 2012; Taberlay et al. 2014). Recent studies have used these features to predict enhancer-gene interactions (Aran and Hellman 2013, 2014; Aran et al. 2013; Andersson et al. 2014; He et al. 2014); however, these methods provide limited insight into the roles of enhancers in cancer, in particular, the transcriptional consequences of abnormal enhancer methylation.

\footnotetext{
${ }^{4}$ These authors contributed equally to this work.

${ }^{5}$ Present address: Institute for Genomic Medicine, Columbia University Medical Center, New York, NY 10032, USA Corresponding author: carmitlevy@post.tau.ac.il

Article published online before print. Article, supplemental material, and publication date are at http://www.genome.org/cgi/doi/10.1101/gr.197194.115.
}

Cancer plasticity refers to the ability of tumor cells to transition between states and evolve under selective pressure, facilitating metastagenesis (Friedl and Alexander 2011; Tam and Weinberg 2013). Tumor heterogeneity and the microenvironment clearly impact cancer cell plasticity (Friedl and Alexander 2011; Brabletz 2012; Meacham and Morrison 2013). However, unlike genetic mutations, DNA methylation is a reversible modification (Franchini et al. 2012); thus, we hypothesized that methylation changes could promote cancer progression by affecting cancer cell plasticity. To test this hypothesis, we extended the analysis of Aran and Hellman $(2013,2014)$ and Aran et al. (2013) and analyzed differential DNA methylation patterns in 25 cancer types and seven sites of metastatic melanoma. Our analysis suggests that enhancer methylation changes can be indicative of patient outcome and may contribute to cancer progression through cancer cell plasticity.

\section{Results}

Enhancers exhibit highly dynamic methylation patterns upon malignant transformation

In order to methodically examine DNA methylation changes involved in malignant transformation, we analyzed 23 distinct

(c) 2016 Bell et al. This article is distributed exclusively by Cold Spring Harbor Laboratory Press for the first six months after the full-issue publication date (see http://genome.cshlp.org/site/misc/terms.xhtml). After six months, it is available under a Creative Commons License (Attribution-NonCommercial 4.0 International), as described at http://creativecommons.org/licenses/by-nc/4.0/. 
cancer types (overall 25 data sets). We used the genome-wide scale Illumina HumanMethylation450 arrays (Dedeurwaerder et al. 2011) to identify differentially methylated regions (DMRs) by analyzing over 6200 DNA methylation profiles of patients' tumors and normal tissues (Supplemental Table S1). We identified 123,649 DMRs (minimum region-wise mean methylation difference $>0.3$, false discovery rate, $q<0.2$ ). Interestingly, we found that enhancers had more DMRs than any other genomic region; promoters and CpG islands exhibited the least variation (Fig. 1A). This result suggests that alterations of enhancer methylation have a significant role in cancer progression, in support of data reported previously (Aran and Hellman 2013, 2014; Ziller et al. 2013; Taberlay et al. 2014). To test whether the selected threshold of 0.3 for differential methylation represented an optimal measure, we calculated the fraction of DMRs in each genomic feature using higher and lower thresholds (from 0.2 to 0.5 ); enhancers were consistently the most variable regions (Supplemental Fig. S1A). As the frequency of $\mathrm{CpG}$ dinucleotides varies with genomic features (Supplemental Fig. S1B), we repeated the differential methylation analysis using methylation levels of individual CpG sites in the array (Supplemental Fig. S1C) and observed consistent results, namely that CpG methylation within enhancers is the most variable compared to all other genomic features.

Given the parallels between embryonic development and oncogenic transformation (Hon et al. 2013), we examined the binding patterns of central pluripotent transcription factors (TFs) POU5F1, SOX2, and NANOG in various DMRs. We observed that multiple binding occurred more frequently at differentially methylated enhancers than at promoters or CpG islands (Fig. 1B). These results support previous findings (Göke et al. 2011; Whyte et al. 2013) suggesting that eDMRs bound by pluripotent TFs may mediate central expression programs. To qualitatively examine the altered enhancers, we analyzed their ChromHMM states (Ernst and Kellis 2012). We found that, compared to enhancers that were not differentially methylated (static enhancers), eDMRs were significantly enriched for the ChromHMM state of "strong enhancers" $\left(P<2 \times 10^{-16}\right)$ (Fig. 1C), similar to previous reports (Aran et al. 2013). These results suggest that enhancers altered in cancer may regulate their target genes more strongly than do unaltered enhancers. It is known that promoter and $\mathrm{CpG}$ island hypermethylation are associated with gene silencing in cancers (Bergman and Cedar 2013), and, indeed, we found that these regions were frequently hypermethylated (63\% and $94 \%$, respectively). In comparison, enhancers were mostly differentially hypomethylated (67\%) (Fig. 1D). Next, we used chromatin marks to qualitatively determine the chromatin features of the hypermethylated and hypomethylated enhancers. Using data from the ENCODE Project (Rosenbloom et al. 2013), we found that differentially hypomethylated enhancers exhibited significantly higher levels of chromatin marks of active DNA (increased DNase I, H3K4me1, H3K4me2, H3K27ac, H2A.Z, EP300, POL2, and decreased CTCF). Differentially hypermethylated enhancers showed the footprints of closed and inactive DNA (Fig. 1E; Supplemental Fig. S1D,E), consistent with previous results (Zhou et al. 2011; Aran et al. 2013; Lam et al. 2014). Taken together, the analysis of multiple tumor types strongly suggests that alteration of the enhancer methylome is a frequent feature of transformed cells and that it 
generally leads to chromatin activation, likely involved in cancer progression.

\section{Differential methylation patterns of eDMRs clusters tumors} according to their organ system

Next, we compared the patterns of methylation change in enhancers between the various types of cancer. Since enhancers show tissue-specific patterns of histone modifications and TF binding in normal cells (Bulger and Groudine 2011), we expected that alter- ation of methylation patterns would be cancer-/tissue-specific. Indeed, most eDMRs (54\%) were unique to a single cancer type (Fig. 2A). Strikingly, principal components analysis (PCA) of eDMRs showed a higher order clustering of cancers into groups related to the same organ system (Fig. 2B). For example, the central nervous system cluster, which includes cancers arising from glia and astrocytes (Fig. 2B, green circle); similarly, the reproductive system cluster consists of breast, uterine, and prostate tumors (Fig. 2B, pink circle). Importantly, PCA of differentially methylated $\mathrm{CpG}$ islands, promoters, and intergenic regions showed no such
A

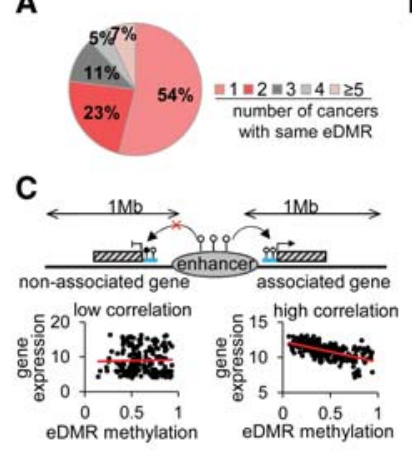

B

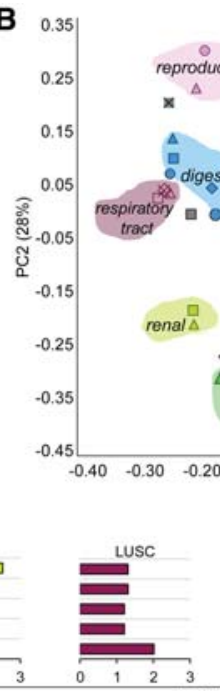

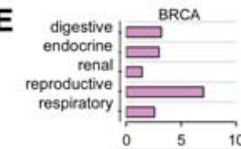
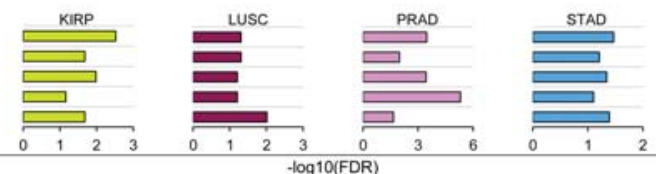

- COAD1 $\triangle$ GBM1

COAD2 $\diamond$ GBM2

$\triangle$ READ $\square$ LGG

$\triangle$ STAD O PA

- LIVER $\triangle$ BRCA

$\triangle$ HNSC $\square$ PRAD

$\triangle$ OSCC O UCEC

$\square$ LUAD $\triangle K I R C$

$X$ LUSC $\square$ KIRP

$\triangle$ BCELL X BLCA

$\triangle \mathrm{MOS}+\mathrm{CHOL}$

$\triangle$ MDS \& CHOL

$\begin{aligned} \triangle \text { THYM } & \square \text { SKCM } \\ & \diamond \text { THCA }\end{aligned}$
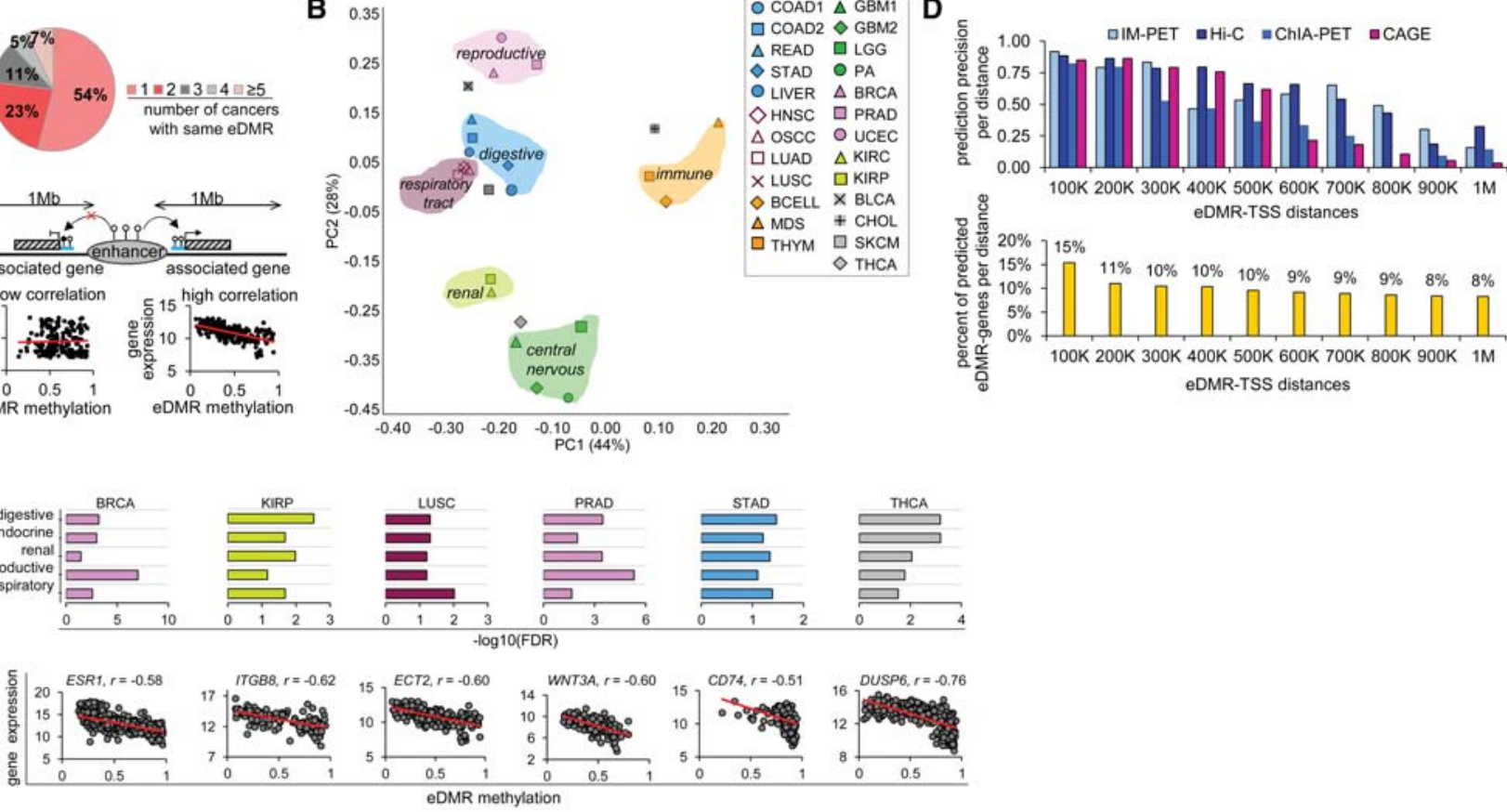

G
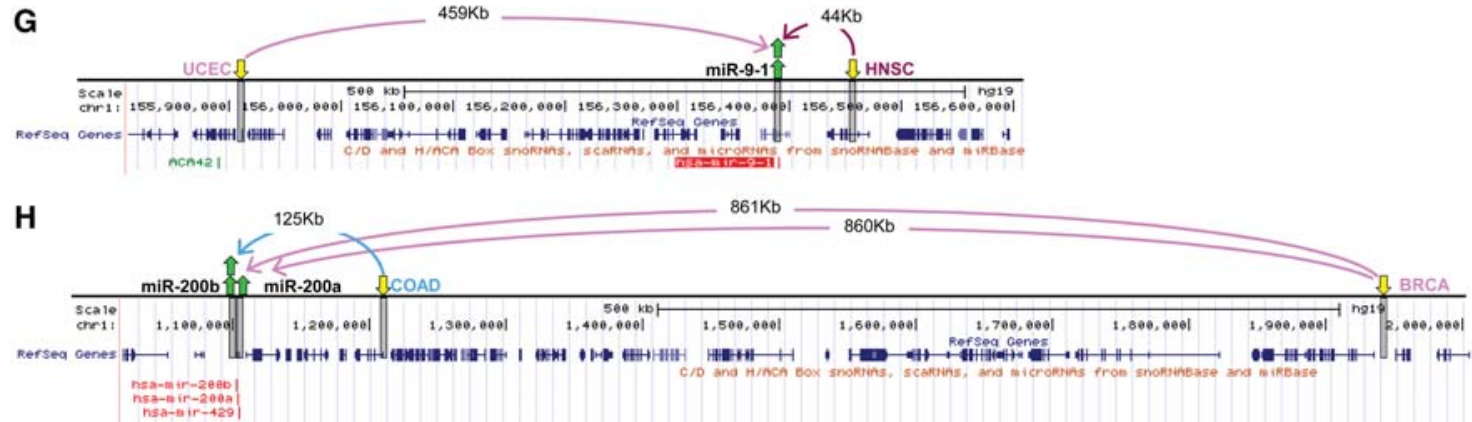

Figure 2. Variation in enhancer methylation classifies primary tumors according to their organ systems. $(A)$ Pie chart shows that most eDMRs are unique (77\% of the eDMRs appear in only one or two cancers, excluding the same cancer type, e.g., GBM1 and GBM2). (B) Principal components analysis (PCA) shows that differential methylation patterns of eDMRs are similar for cancers belonging to the same organ systems (circled and colored similarly). First and second principal components ( $x$-axis: PC1; $y$-axis: PC2) account for $72 \%$ of variation (see Supplemental Fig. S2A-C for PCA analysis of methylation changes within CpG islands, promoters, and intergenic regions). (C) Schematic of method for identification of eDMR-gene and eDMR-miRNA pairs (see Supplemental Fig. S2H for flow chart). (D) (Top) Precision of eDMR-gene pair predictions is compared to predictions from CAGE (Andersson et al. 2014), IM-PET (Teng et al. 2015), ChIA-PET, and Hi-C (Teng et al. 2015) methods (see text). (Bottom) Percent of predicted eDMR-gene pairs. Results in both plots are divided into groups based on the distances between eDMR and transcription start site (TSS). Most predictions ( $>56 \%$ ) lie within a distance of $<500 \mathrm{~kb}$ (abbreviations: 100K, 0-100,000 bp, 200K: 100,001-200,000 bp, and so forth). (E) eDMR-associated genes are distinctly enriched for genes involved in diseases related to a particular organ system. Disease-related genes were derived from the DISEASES database (see Methods). Scores represent $-\log _{10}$ (FDR-corrected $P$-values). ( $F$ ) Scatter plots (a point for each patient) show examples of tissue-specific genes linked to cancer risk and their eDMRs (the same six cancer types shown in $E$ ). Spearman's correlations between eDMRs methylation and associated-gene expression are shown. (G) Landscape of two differentially hypomethylated eDMRs in uterine (UCEC) and head and neck (HNSC) cancers, associated with a single miRNA, miR-9-1, which is up-regulated in both cancers. $(H)$ Landscape of two differentially hypomethylated eDMRs in breast (BRCA) and colon (COAD) cancers associated with two miRNAs of the same family, miR-200a and miR-200b; each is up-regulated in the respective tumors. In G,H, yellow arrows mark hypomethylated eDMRs, green arrows mark up-regulated miRNAs; gray boxes mark eDMR and miRNA regions in the hg19/GRCh37 genome; distances between miRNAs and eDMRs are indicated on arrows. All parts of the figure refer to eDMRs determined between normal samples and primary tumors. 
clustering (Supplemental Fig. S2A-C). No library preparation batch affect was found either (Supplemental Fig. S2D,E). This evidence provides confidence that our findings were not affected by batch effect and strengthen our hypothesis that enhancer methylation changes may have meaningful biological roles.

Next, in order to uncover the functional effect of altered enhancer methylation, we first compared the genomic neighborhood of eDMRs with static enhancers (those showing no differential methylation). We found that eDMRs were flanked by more genes and microRNAs (miRNAs) than were static enhancers (Supplemental Fig. S2F, G). To identify which genes the eDMRs regulate, we developed an integrated model combining multi-omics data for associating enhancers with their target genes (eDMR-associated genes) (Fig. 2C; Supplemental Fig. S2H for pipeline). Since it is known that there is an inverse correlation between DNA methylation and chromatin activity (Zhou et al. 2011), we retained only inversely correlated eDMR-gene pairs, which represented the majority of our data (Supplemental Fig. S2I). In order to assess the validity of our eDMR-gene pair predictions, we compared our results to other methods that identify enhancer-promotor associations based on physical interactions IM-PET (He et al. 2014), ChIAPET, Hi-C (Teng et al. 2015), and transcriptional activities of interacting enhancer-promoters (cap analysis gene expression; CAGE) (Andersson et al. 2014). Our model predicted eDMR-gene pairs separated by $400 \mathrm{~kb}$ or less at a precision rate of $75 \%$ or better (Fig. 2D), indicating that our model can reliably predict interacting enhancer gene pairs.

Similar to their enhancers (Fig. 2B), the genes predicted to be regulated by the eDMRs also showed organ system patterns of enrichment (Fig. 2E; Supplemental Table S3). Using our model, we were able to identify known tissue-specific and ubiquitous oncogenic genes and microRNAs (Fig. 2F-H), such as: ESR1 in breast cancer (Holst et al. 2012; Aran and Hellman 2014), ECT2 in lung cancer (Murata et al. 2014), and WNT3A in prostate cancer (Verras et al. 2004), hsa-miR-9-1 (Ma et al. 2010) and the clustered miRNAs hsa-miR-200a and miR-200b (Korpal et al. 2008; see Supplemental Table S4 for full list of predicted eDMR-gene pairs). Taken together, our model defines cancer-related eDMRs as organsystem-specific regulators of genes and miRNAs that are central to malignant transformation.

\section{Accumulation of eDMR hypomethylation correlates with likelihood of metastasis}

Our analysis showed that changes in methylation patterns of enhancers in primary tumor tissues are influenced by their relation to the organ system (Fig. 2B). We were curious what would occur to DNA methylation patterns of cells that disseminate from the primary location and colonize in metastatic sites. To evaluate this, we focused on melanoma, a highly metastatic cancer (Braeuer et al. 2014). We compared methylation data from metastatic melanoma patients (seven distinct locations) to data from patients with primary in situ melanoma (Supplemental Table S1). Consistent with our observation of differential methylation patterns in primary tumors (Fig. 1A), the majority of "metastatic DMRs" occurred within enhancers (Fig. 3A), suggesting that enhancers play important roles, not only in malignant transformation but also in metastatic progression. Additionally, metastatic eDMRs differentiate much better between patient outcomes than do DMRs from any other genomic feature (Fig. 3B; Supplemental Fig. S3A). The majority of eDMRs were specific to a single metastatic site (Fig. 3C; Supplemental Fig. S3B) and were mostly (74\%) hypomethylated (Fig. 3D), similar to our observations in primary cancers (see Figs. 1D, 2A). Strikingly, Figure 3E shows that accumulation of hypomethylated enhancers highly correlates with the likelihood of forming metastases at distant organs derived from Meyers and Balch (1998). For example, melanoma is more likely to metastasize to lymph nodes than to the brain; correspondingly, a greater number of enhancers are differentially hypomethylated in brain metastases than in lymph node metastases $(2.24 \%$ and $0.33 \%$, respectively) (Fig. 3E). In contrast, we found no correlation between the fraction of differentially hypermethylated metastatic eDMRs and the frequency of spreading to organs (Supplemental Fig. S3C). These results are in agreement with studies suggesting that global hypomethylation is a common feature of diseased states (Pogribny and Beland 2009). Since widespread DNA methylation changes are associated with aging (Richardson 2003), we calculated the correlations between patient ages and accumulation of enhancer methylation changes. Encouragingly, we found no significant correlation between eDMR methylation changes in metastatic tissues and the patient age (Supplemental Fig. S3D), suggesting that metastatic progression corresponds to cancer-related changes and not age-related changes.

In order to specifically explore whether melanoma eDMRs could promote metastatic growth, we identified genes differentially expressed between primary and metastatic melanoma and compared the correlation of these genes with methylation patterns of differentially methylated enhancers and promoters (eDMRs and pDMRs, respectively). We found that eDMRs were significantly more correlated with differentially expressed genes than were pDMRs (Supplemental Fig. S3E). Next, we divided the genes into two groups: eDMR-associated genes, and all other genes not identified by our model to be associated with eDMRs (control genes) (Fig. 3F), and examined the differential expression patterns of these two groups. The percentage of genes that were differentially expressed between normal and primary melanoma was similar for both groups (60\% and 53\% for eDMR-associated genes and control genes, respectively) (Fig. 3F); however, between primary and metastatic melanoma, the eDMR-associated genes were significantly more variable than the control genes (binomial distribution, $P<2 \times 10^{-16} ; 57 \%$ and 3\%, respectively) (Fig. 3F, marked by gray areas). This observation supports our notion that a functional interaction exists between the eDMRs and their predicted associated genes. Additionally, we note that a fraction of the control genes were down-regulated in both primary and metastatic melanoma (Fig. 3F, left panel, marked with dashed box). This group was enriched $(q<0.01$, FDR corrected) with Gene Ontology (GO) terms associated with tissue development (GO:0008544, GO:0007398) and differentiation (GO:0030216, GO:0009913, GO:0030855), likely a feature of tumor de-differentiation (Brabletz 2012).

\section{Methylation plasticity of melanoma eDMRs is associated with increased patient mortality}

The transition of cancer from the in situ primary stage to the metastatic stage involves phenotypic plasticity (Craene and Berx 2013), which facilitates migration through tissues and adaptation to changing microenvironments. Underlying tumor plasticity are genetic and epigenetic regulatory layers that are reprogrammed in the context of cancer (Friedl and Alexander 2011). Interestingly, we found that $18 \%(N=277)$ of melanoma metastatic eDMRs $(N=$ 1539) switched the direction of differential methylation as melanoma progressed (denoted "switched" eDMRs) (Fig. 4A, bottom circle). For example, regions that were hypermethylated

\section{Genome Research}

www.genome.org 
A

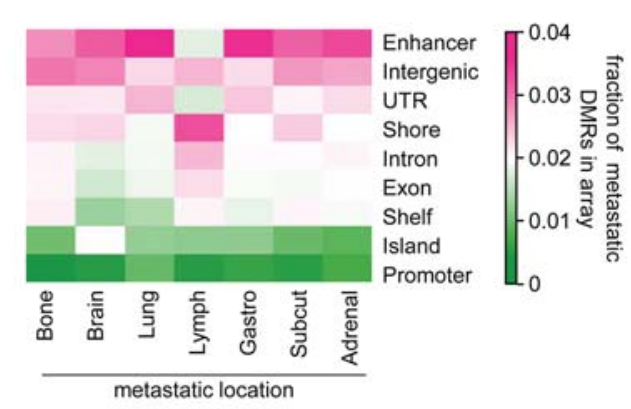

B

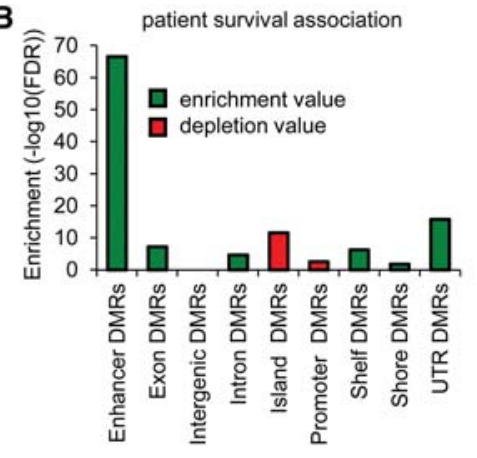

C

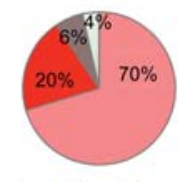

$\square 1=2 \quad 3 \quad \square \geq 4$

number of metastatic locations sharing sam
metastatic eDMR

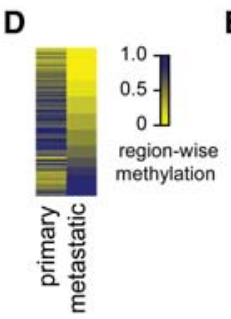

E

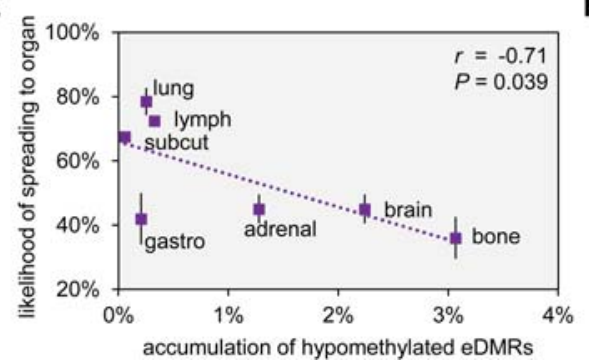

$\mathbf{F}$

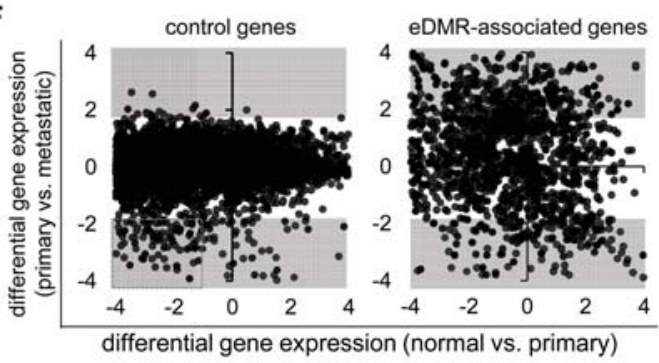

Figure 3. Accumulation of eDMR hypomethylation correlates with likelihood of metastasis and patient outcome. (A) Heat map shows that enhancers are more differentially methylated than other genomic features ( $y$-axis; sorted according to fraction of DMRs in each category) (see Fig. 1A). (Adrenal) Metastases to adrenal glands, (gastro) metastases to the gastrointestinal tract, (lymph) metastases to lymph nodes, (subcut) subcutaneous metastases. (B) Enhancers are significantly more enriched with DMRs that can differentiate between patient survival times (see Methods). Green and red bars represent enrichment and depletion, respectively. $Y$-axis represents the $-\log _{10}$ (FDR-corrected $P$-values) significance (binomial distribution). (C) Most melanoma eDMRs (70\%) are exclusive to one metastatic site (see Fig. 2A). (D) Heat map shows that most eDMRs are hypomethylated between primary and metastatic melanoma (74\%). (E) Accumulation of eDMR hypomethylation is negatively correlated with the likelihood of forming melanoma metastases at each body location (Pearson's correlation, $r=-0.71, P=0.039$, one-sided hypothesis testing). $(F)$ Compared to all other genes (control genes; $y$-axis, left panel), genes associated with eDMRs ( $y$-axis, right panel) are differentially expressed between primary melanoma and metastatic melanoma ( $57 \%$ and $3 \%$, respectively; marked by gray areas), whereas, differential expression between melanocytes and primary melanoma is similar for both groups (control genes, $53 \%$, left panel; eDMR-associated genes, $60 \%$, right panel; $x$-axes). All parts of the figure refer to differential methylation between patients with primary melanoma and patients with metastatic melanoma.

between normal melanocytes and primary melanoma were hypomethylated between primary and metastatic melanoma. We also defined two other eDMR groups: "consistent" and "de novo." The "consistent" eDMRs exhibited differential methylation changes in the same direction (e.g., hypomethylated between normal melanocytes and primary melanoma, and hypomethylated between primary and metastatic melanoma) (Fig. 4A, right circle). The "de novo" group exhibited differential methylation only between primary and metastatic melanoma samples and not between normal melanocytes and primary tumors (Fig. 4A, left circle). We examined whether these three groups of eDMRs differed with respect to metastatic progression by comparing their ability to correlate with survival of patients (see Methods). To our knowledge, this is one of the first examples (Stone et al. 2015) of a survival analysis applied to DNA methylation patterns of enhancers. We identified 30 eDMRs that were associated with patient survival rates (marked "survival" eDMRs) (Fig. 4A, middle circle). Remarkably, these survival eDMRs were enriched with the switched eDMRs $\left(P=9.4 \times 10^{-5}\right)$ (Fig. 4A, bottom circle), whereas de novo eDMRs were significantly depleted $\left(P=1.6 \times 10^{-2}\right)$ (Fig. 4A, left circle). In addition, the switched eDMRs exhibited the highest conservation scores (Fig. 4B) and the fewest copy number variations (CNVs) in melanoma patients (Fig. 4C). These results suggest that eDMRs, in particular the switched eDMRs that exhibit methylation plasticity, are functionally important in cancer.
Given these results, we hypothesized that methylation plasticity may play important roles in melanoma metastatic progression. To examine this, we performed survival analyses also on the eDMR-associated genes, using their expression patterns across patients. Remarkably, we found that $40 \%$ were able to significantly differentiate between survival outcomes $\left(\chi^{2}, q<0.1\right.$, FDR corrected); these genes include: ATP2B1 (Lee et al. 2002), FMNL2 (Zhu et al. 2008), KIT (Tian et al. 1999), PRKCE (Sharif and Sharif 1999), and VGF (Mitra et al. 2008). Figure $4 D$ represents such an example in which the oncogene KIT and the eDMR (located at Chr4:55708295-55709294, hg19/GRCh37) not only exhibit switched patterns of methylation and expression, but both independently distinguish between patient survival rates based on their expression and methylation patterns, respectively (Fig. 4D, left and right panels). The KIT promoter was not differentially methylated, nor did the methylation pattern differentiate between survival times (Fig. 4D, middle panel).

To experimentally test the role of enhancer methylation on the transcriptional regulation of KIT, we first examined KIT expression upon treatment with 5-aza-2'-deoxycytidine (5-aza-dC), a DNA methyltransferase inhibitor. There was a significant increase in KIT levels in treated compared with untreated cells (Fig. 4E). Next, we cloned the KIT enhancer upstream of a luciferase reporter and demonstrated that its expression was reduced upon enhancer methylation (Fig. 4F; Supplemental Fig. S4A). Finally, we examined the role of KIT up-regulation on the invasive potential of 
A

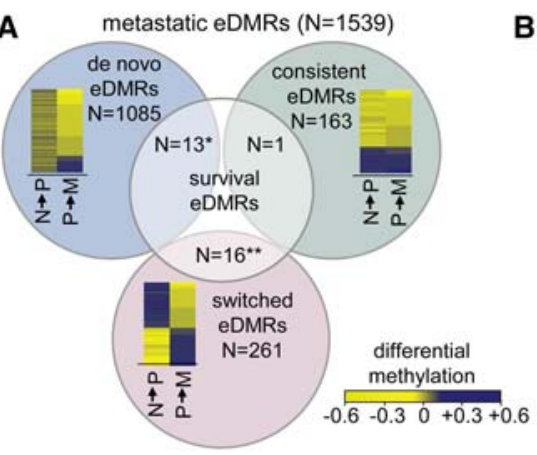

D
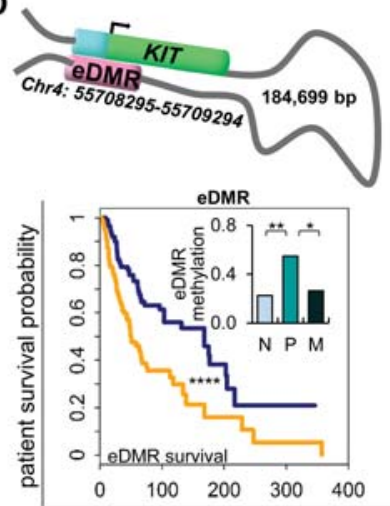

B

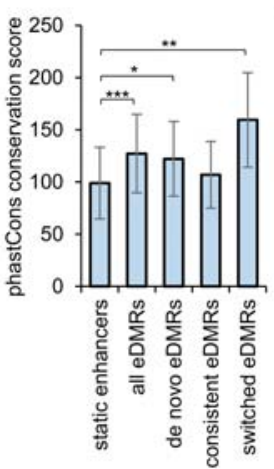

c

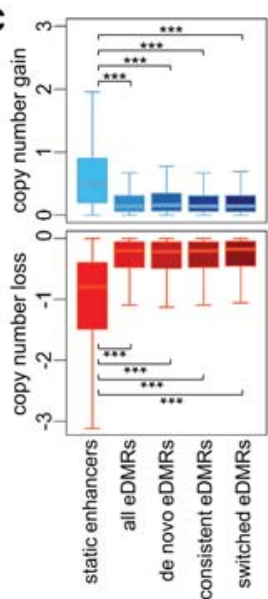

high methylation = low methylation KIT promoter

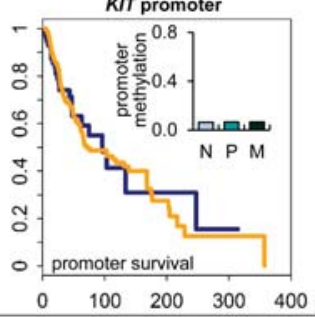
overall survival (months) low expression -

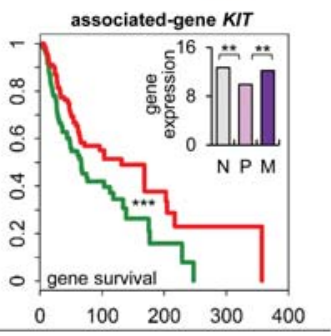
$\begin{array}{lllll}0 & 100 \quad 200 & 300 \quad 400\end{array}$ high expression =

F

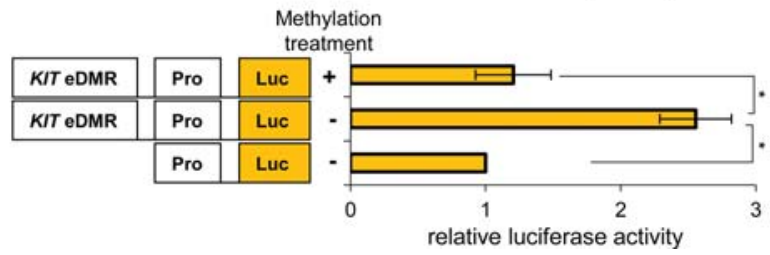

E

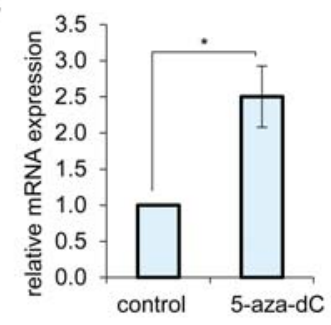

G

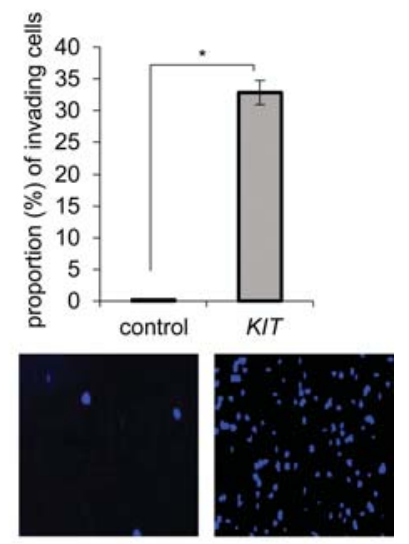

Figure 4. Methylation plasticity of melanoma eDMRs is associated with patient mortality. $(A)$ Venn diagram of three types of eDMRs found in metastatic melanoma: "de novo," "consistent," and "switched" (described in text). Heat maps display differential methylation between normal melanocytes and primary melanoma $(\mathrm{N} \rightarrow \mathrm{P})$, and between primary and metastatic melanoma $(\mathrm{P} \rightarrow \mathrm{M})$. "Switched" eDMRs are enriched for "survival" eDMRs and "de novo" eDMRs are depleted, hypergeometric distribution; $\left(^{* *}\right) P=9.4 \times 10^{-5},\left(^{*}\right) P=1.6 \times 10^{-2}$, respectively. Numbers represent amount of eDMRs in each category. $(B)$ PhastCons conservation scores of enhancers' sequence conservation, switched eDMRs exhibit the highest sequence conservation (see Methods) compared to all other enhancers. Two-way ANOVA analysis; ( $\left.{ }^{*}\right) P=4.2 \times 10^{-6},\left({ }^{* *}\right) P=9.9 \times 10^{-10},\left({ }^{* * *}\right) P=2.9 \times 10^{-11} ;$ bars represent average sequence conservation, error bars represent standard deviation. (C) Copy number variations (CNVs) are infrequent within eDMRs compared to static enhancers. Two-way ANOVA analysis; $\left({ }^{* *}\right) P<2 \times 10^{-16}$. (D) (Top) Schematic representation of the eDMR-gene pair: Chr4:55708295-55709294 and KIT gene. The eDMR (pink) is interacting with the promoter (cyan) of KIT gene (green) through chromatin looping. The distance between the eDMR and the transcription start site (TSS) is noted in the schematic. (Bottom, left) Kaplan-Meier survival plot shows significant differences between survival times of patients based on the methylation levels of the eDMR of the gene KIT $\left.\left(\chi^{2},{ }^{* * * *}\right] P=2.9 \times 10^{-4}\right)$. Patients were divided into two groups of higher (blue) and lower (yellow) methylation levels compared to the median (see Methods). (Middle) Plot shows that patients with higher or lower methylation of the KIT promoter show no significant difference in survival rates. (Right) Plot shows significant differences between survival times of patients with higher (green) and lower (red) expression levels of $\left.K I T\left(\chi^{2},{ }^{* * *}\right] P=2.4 \times 10^{-3}\right)$. (Insets, left and right) Both eDMR methylation and KIT expression exhibit switched patterns between normal melanocytes $(\mathrm{N})$, primary melanoma $(\mathrm{P})$, and metastatic melanoma (M). (Middle inset) KIT promoter does not exhibit switched methylation patterns. $\left(^{*}\right) q<0.05,\left({ }^{* *}\right) q<0.01$. (E) Inhibition of DNA methylation increases KIT expression. WM3682 melanoma cells were treated with 10 $\mu \mathrm{m}$ 5-aza-dC or DMSO (control); KIT mRNA expression was normalized to levels of GAPDH. Data are relative to levels in control-treated cells. Error bars represent \pm SEM; $\left(^{*}\right) P<0.05 ; N=3$. $(F)$ WM3682 melanoma cells were transfected with methylated KIT-eDMR reporter plasmid, unmethylated KITeDMR reporter, or reporter without eDMR (control). Firefly luciferase activity was normalized to Renilla luciferase activity. Fold-changes are relative to control. Error bars represent $\pm \mathrm{SEM} ;\left(^{*}\right) P<0.05 ; N=3$. (G) (Top) KIT overexpression increases melanoma invasiveness. WM3682 melanoma cells were transfected with KIT expression vector or empty vector (control); invasion ability was analyzed. Error bars represent $\pm \mathrm{SEM}$; $\left.{ }^{*}\right)$ P $<0.05 ; \mathrm{N}=3$. (Bottom) A representative image of invading cells.

melanoma cells (Fig. 4D). Remarkably, noninvasive melanoma cells (Golan et al. 2015) acquired significant invasion ability upon KIT overexpression (Fig. 4G; Supplemental Fig. 4B). Taken together, our data demonstrate that enhancer methylation contributes to cancer progression by directly regulating oncogene expression.

Next, we asked whether the dynamics of eDMR methylation and eDMR-associated genes expression are a result of tumor het- erogeneity, evolution of the disseminated cancer cells, or are induced by the metastatic niche. To assess this, we selected two eDMR-associated genes, CTYL1 and KIF14, which were differentially expressed only between primary and metastatic melanoma (not between normal and primary melanoma tissues). CYTL1 is a cytokine-like protein implicated in lung cancer and neuroblastoma (Kwon et al. 2012; Wen et al. 2012), and KIF14 is an oncogene 
essential for cytokinesis (Corson et al. 2005); both are up-regulated in melanoma bone metastases $(P<0.05)$ (Fig. 5A, top and bottom panels). High expression levels of these genes is correlated with poor patient survival rates ( $\chi^{2} q<0.1$, FDR corrected) (Fig. 5A, green stepwise curves). Enhancers of these genes were differentially methylated, yet their promoters were not (Fig. 5A, right and middle, panels, respectively). To experimentally analyze the role of enhancer methylation on the transcriptional regulation of CYTL1 and KIF14, we first examined whether their expression is sensitive to DNA methylation. CYTL1 and KIF14 expression was significantly up-regulated upon treatment of cells with 5-aza-dC (Fig. $5 \mathrm{~B})$. Next, we cloned the identified enhancers of CYTL1 and KIF14 upstream of a luciferase reporter and observed a decrease in the reporter expression upon enhancer methylation (Fig. 5C; Supplemental Fig. S4A). These results strengthen the validity of our model by demonstrating that the identified eDMRs elicit a response to changes in their methylation and directly regulate the expression of their associated genes: KIT, CYTL1, and KIF14.
Finally, to assess changes in gene expression induced by metastatic melanoma cells colonization in the bone tissue, we first generated a melanoma cell line stably expressing the GFP gene to enable tracking of the melanoma cells. Next, we established a coculture of melanoma cells with human primary osteoblasts (Fig. 5D; Dillon et al. 2012) and found that levels of CYTL1 and KIF14 were significantly increased compared to their levels in melanoma cells cultured alone (Fig. 5E). Our data support the notion that homing into a new microenvironment induces expression of procancer genes, which are regulated by eDMRs in melanoma metastatic cells. Taken together, our data suggest that methylation changes at enhancers contribute to melanoma phenotypic plasticity and ultimately to the patient's chance of survival.

\section{Discussion}

Here, we performed an analysis of DNA methylation alterations in over 6200 cancer patients from 31 cancer data sets, including
A

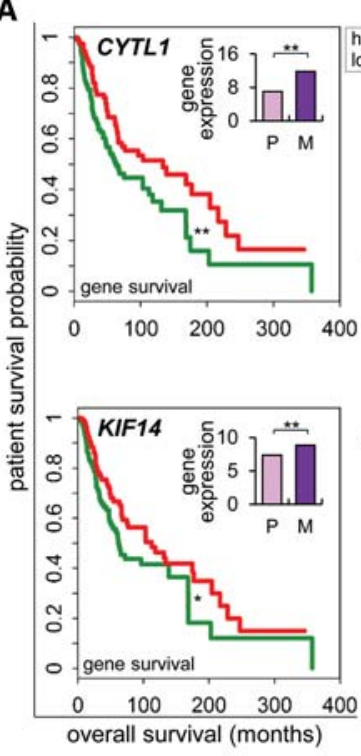

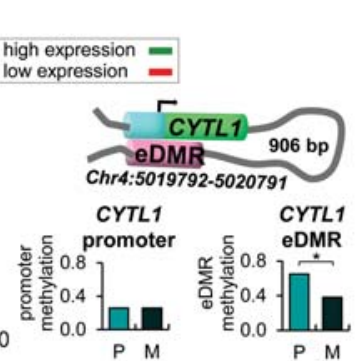

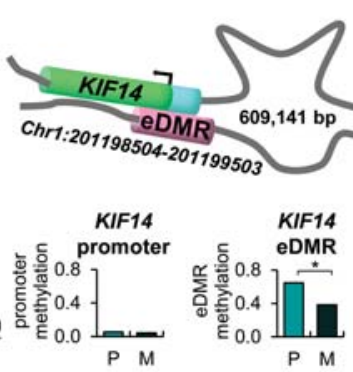

B

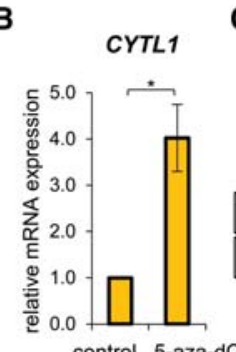

C
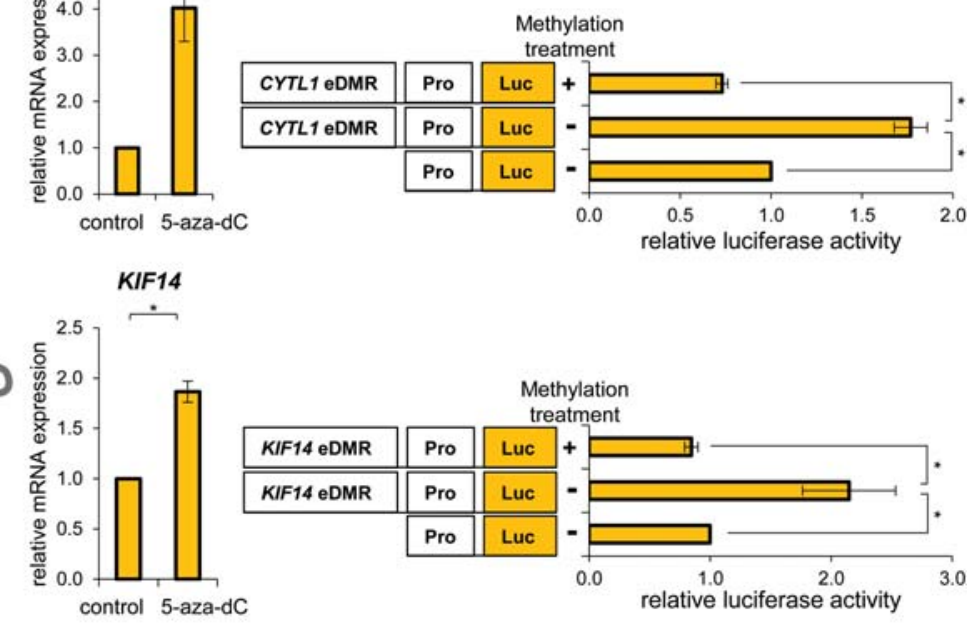

$\stackrel{5 \text { days }}{\longrightarrow}$

Melanoma cells separation $\longrightarrow$ Gene expression analysis

E

relative gene expression

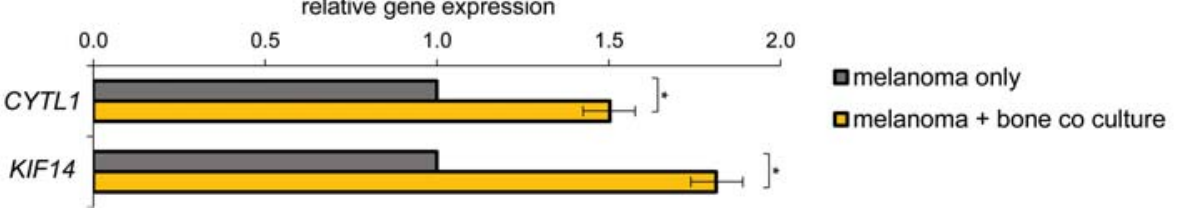

Figure 5. Melanoma coculture with osteoblasts alters pro-cancer eDMR-associated gene expression. $(A)$ Schematic representation of the eDMR-gene pairs: Chr4:5019792-5020791 for CYTL1 and Chr1:201198504-201199503 for KIF14. The eDMR (pink) is interacting with the promoter (cyan) of the gene (green) through chromatin looping. Kaplan-Meier survival plots (left panels) show significant differences between patient outcomes at high and low expression levels (green and red curves, respectively) of CYTL1 and KIF14 genes. (Insets) Bar graphs show up-regulation of CYTL1 and KIF14 in melanoma bone metastases. Middle panels: Methylation of the promoters of these genes does not vary between primary melanoma (P) and melanoma bone metastases (M). Right panels: Enhancers are differentially hypomethylated in melanoma bone metastases (Wilcoxon rank-sum tests, [*] $q<0.15$, $\left[{ }^{* *}\right] q<0.05$; FDR adjusted). (B) WM3682 melanoma cells were treated with $10 \mu \mathrm{m} 5$-aza-dC or with DMSO (control) followed by quantification of CYTL1 and KIF1 4 mRNA levels that were normalized to GAPDH. Data are relative to levels in control-treated cells. Error bars represent $\pm S E M ;\left({ }^{*}\right) P<0.05 ; N=3$. (C) WM3682 melanoma cells were transfected with methylated CYTL1-eDMR or KIF14-eDMR reporter plasmids, unmethylated eDMR reporter, or reporter without eDMR (control). Firefly luciferase activity is normalized to Renilla luciferase. Fold-changes are relative to control. Error bars represent \pm SEM; $\left(^{*}\right)$ $P<0.05 ; N=3$. (D) Experimental design scheme. (E) Levels of CYTL1 and KIF14 mRNA were determined in melanoma cells before and after coculturing with osteoblasts. Data were normalized to levels of actin. Error bars represent \pm SEM; $\left(^{*}\right) P<0.05 ; N=3$. 
23 diverse primary malignant tumors, two benign tumors, and melanoma metastases to seven distinct organs. This extensive analysis revealed that most methylation variation occurs at enhancers (Figs. 1A, 3A; Supplemental Fig. S1C). Changes in methylation patterns could result from competition between methylation and de-methylation processes or from errors in replication (Jones 2012). Our findings support the latter hypothesis, for two reasons. First, replication-related methylation errors occur more frequently in methylated regions, such as enhancers, where replication errors lead to loss of methylation; this is what we observe (Fig. 1D). Second, expression of DNMT3B, which encodes a methylase enzyme, was up-regulated in many tumors, and hence, we would expect increased methylation since we observed no significant differences in expression of TET genes, which encode demethylase enzymes (Supplemental Fig. S2J). However, since the majority of changes in enhancers' methylation involves de-methylation, we hypothesize that loss of enhancer methylation could have occurred during replication.

Enhancers play central roles in normal development and differentiation by responding to complex environmental cues. Cancer cells are exposed to changing environmental conditions that require their adaptation; this mainly occurs through epigenetic reprogramming (Friedl and Alexander 2011; Goding et al. 2014). We hypothesize that, in the context of cancer, enhancer methylation may be primed to respond to microenvironmental signals. To test this, we mimicked the microenvironment of melanoma bone metastases and recapitulated the perturbation of expression of eDMR-associated genes that were altered in the bone metastases. These results suggest that the metastatic niche can alter expression of eDMR-associated genes.

Interestingly, we show that, in comparison to DMRs in other genomic features (CpG islands, promoter, exons, introns, etc.), eDMRs can differentiate best between patient outcomes (Supplemental Fig. S4D). These results demonstrate the important role of alteration of enhancer methylation in cancer progression. However, we do not exclude that other factors may drive cancer progression, nor are we suggesting a direct causality between enhancer methylation and cancer progression; it may well be that alteration of enhancer methylation is a secondary event of the malignancy.

Nevertheless, we did observe that methylation patterns of eDMRs may be informative of patient survival rates (Fig. 3B). Within this group was a subset (18\%) of highly conserved eDMRs that displayed methylation plasticity ("switched" eDMRs) (Fig. 4A-C) and provided insight into alteration of their associated genes (Fig. 4D). This is one of the first studies (Stone et al. 2015) to suggest that methylation patterns of enhancers can be used to predict patient outcome. Moreover, it has been shown that, in many diseases, including cancer, methylation changes are accumulative as the cancer progresses (Pogribny and Beland 2009); here, we show that methylation plasticity may also play important roles in cancer progression (see Supplemental Fig. S4C for model of methylation plasticity and cancer progression). A prime example of the relationships between metastatic progression, methylation plasticity, and patient mortality is that of the eDMR-associated oncogene, KIT (Fig. 4D-F). Both the methylation of the eDMR and the expression of the KIT display plasticity (Fig. 4D) and significantly distinguish between patient survival rates. Our results suggest that enhancer methylation patterns may be informative of patient outcomes and that they may influence malignant progression via methylome plasticity.

\section{Methods}

\section{TCGA and GEO data sources: DNA methylation, RNA-seq, smRNA-seq}

Publicly available data of DNA methylation, RNA-seq gene expression, and small RNA-seq miRNA expression from cancer patient tissues were obtained from The Cancer Genome Atlas (TCGA, https://tcga-data.nci.nih.gov/tcga) and from the National Center for Biotechnology Information (NCBI) Gene Expression Omnibus (GEO) repository (http://www.ncbi.nlm.nih.gov/gds). See Supplemental Table S1 data set information.

\section{Chromatin marks, transcription factors, sequence conservation, and copy-number variation data}

For analysis of chromatin marks (Fig. 1E; Supplemental Fig. 1D,E), we downloaded RNA polymerase II (POL2), histone modifications H2A.Z, H3K4me1, H3K4me2, and H3K27ac, histone acetyltransferase (EP300), CCCTC-binding factor (CTCF), DNase I hypersensitivity, and Chromatin State Segmentation by HMM (ChromHMM) from the ENCODE Project. Replicate experiments were averaged. See Supplemental Materials for URL and description and Supplemental Table S2 for ENCODE Project cell types.

For the transcription factor analysis (Fig. 1B), we downloaded processed ChIP-seq data of POU5F1, SOX2, and NANOG, downloaded from NCBI/GEO (GSE46130). Replicate experiments were averaged. The TF peaks were overlapped with the coordinates of the differentially methylated enhancers, promoters, and CpG islands.

For the conservation analysis of enhancer (Fig. 4B), we used 100-way PhastCons conservation data.

Copy number variations for skin cutaneous melanoma (SKCM) were obtained from TCGA database as genomic segments after removal of germline CNV (TCGA_SKCM GSNP6noCNV_gSeg).

\section{Defining differentially methylated regions}

First, we matched the CpG codes in the Illumina HumanMethylation450 microarray to their genomic coordinates using the microarray platform (NCBI/GEO record GPL13534; human genome release hg19/GRCh37). Second, we annotated the CpGs using the information in this record, identifying CpG sites belonging to $\mathrm{CpG}$ islands, shores, shelves, enhancers, and UTRs. Given that this record does not have information of $\mathrm{CpG}$ sites in promoters, exons, or introns, we used the UCSC table "knownGene" to identify these sites. Third, some CpG sites had several annotations; thus, we divided the CpGs into unique (nonoverlapping) genomic features using the following prioritization: (1) promoters, (2) CpG islands, (3) enhancers, (4) introns, (5) exons, (6) UTRs, (7) shores, (8) shelves, and (9) intergenic regions. Fourth, for each separate genomic feature, we constructed intervals using a window of $500 \mathrm{bp}$ directly upstream of and downstream from the CpG coordinate. Overlapping intervals (same genomic feature) were joined, and extended into a larger interval. Sixtynine percent of the regions had a length of $1000 \mathrm{bp}, 28 \%$ a length $>1000 \mathrm{bp}$ and $<2000 \mathrm{bp}$, and no region was greater in length than $7500 \mathrm{bp}$. Fifth, we used these genomic intervals to calculate regionwise methylation levels based on the average methylation of all CpG sites within the interval; we performed this for all normal and all tumor samples in each cancer data set. Sixth, we used the two-sample Wilcoxon test to identify differentially methylated regions between normal and primary samples (methylation threshold $>0.3, q<0.2$, FDR corrected); metastatic DMRs were identified by comparing primary and metastatic melanoma (methylation threshold $>0.2$ and $q<0.2$ ). The fractions of DMRs in each

\section{Genome Research}

www.genome.org 
genomic feature presented in Figures $1 \mathrm{~A}$ and $3 \mathrm{~A}$ and Supplemental Figure S1C were normalized to the amount of intervals in each genomic feature in the Illumina HumanMethylation450 array (Dedeurwaerder et al. 2011).

\section{Gene and miRNA differential expression analysis}

A fold-change of 1.25 was used to determine differentially expressed genes and miRNAs $(q<0.05$, FDR corrected) (see Supplemental Methods for description).

\section{Model for prediction of gene-eDMR associations}

We developed an integrated model to predict genes or miRNAs regulated by eDMRs (Fig. 2C; flowchart in Supplemental Fig. S2H). First, we calculated the differential methylation in enhancer and promoter regions, retaining the eDMRs, and the level of methylation change in promoters, which were used in the analysis of the genes (see third step). Second, we computed Spearman's correlations between eDMR methylation patterns and gene/miRNA expression patterns across the same (matched) patients. Highly correlated eDMR-genes or eDMR-miRNAs pairs were retained (correlation below -0.4). Third, we filtered out genes whose promoters had a differential methylation greater than 0.2, enriching for genes with expression change due to enhancer, rather than by promoter, methylation variability (performed only for predicting eDMR-associated genes and not associated-miRNAs, since annotation for miRNA promoters is incomplete). Fourth, we selected eDMR-gene and eDMR-miRNA pairs located on the same chromosome, with a maximal linear distance of $1 \mathrm{Mbp}$ between the center of the enhancer and the transcription start site (for genes) or premiRNA start position (for miRNAs). Fifth, we ranked eDMR-gene predictions (see Supplemental Table S4 and subsection "Ranking eDMR-gene pairs predictions").

\section{Ranking eDMR-gene pairs predictions}

In order to provide confidence that the predicted eDMR-gene pairs represent active enhancer-gene interactions, we applied an integrated scoring function. Our scoring scheme combined quantitative and qualitative features of the eDMR-gene pairs. First, we ranked each eDMR-gene pair based on the following quantitative features: (1) Spearman's correlation between the eDMR and gene; (2) greater enhancer differential methylation; (3) greater gene expression fold-change; and (4) shorter eDMR-gene distances. Second, we used qualitative features which could increase ranking if existing: (1) eDMR overlap with H3K4me1, H2A.Z, or DNase I peaks; and (2) promoter overlap with $\mathrm{H} 3 \mathrm{~K} 4 \mathrm{me} 3$ or DNase I peaks. We summed up the scores for each eDMR-gene pair (ties were given an average ranking score) and sorted them based on their overall score, yielding ranked predictions (Supplemental Table S4).

\section{Gene enrichment analysis for diseases}

We used the DISEASES website (http://diseases.jensenlab.org) (Pletscher-Frankild et al. 2015) to evaluate enrichment for diseases in particular organ systems (Fig. 2E). Diseases were identified using keywords: digestive (digest-, gastro-, gastric, stomach), endocrine (endocrine-, gland disease, gland cancer, gland neoplasm, thyroid, pancreas), renal (kidney, renal, nephron-, nephri-), reproductive (reproduct-, breast, prostate, uterine, cervic-, cervix, uterus), respiratory (lung, respirator); duplicate genes were removed to ensure unique values for hypergeometric distribution significance testing and FDR corrected.

\section{Survival analysis}

Survival time was derived from the "overall survival" column of the clinical data files obtained for TCGA samples. First, patients were divided into two groups (high and low) by comparing the eDMR methylation level to the median methylation of the eDMRs across all patients. Similarly, for gene expression survival analysis, patients were divided into two groups by comparing the gene expression of each patient to the median expression of all patients. Significant differences between the two groups were determined using the $\chi^{2}$ distribution $(q<0.1$, FDR corrected).

Enrichment for association of DMRs with patient survival (Fig. 3B) was determined by comparing the amount of DMRs that can significantly differentiate between patient outcomes with the amount of static regions that can do the same (determined separately for each genomic feature).

\section{Multiple testing}

All significance tests were corrected for false discovery rates (FDR) using the Benjamini and Hochberg adjustment (Benjamini and Hochberg 1995).

\section{Computational data analysis}

Data analyses were performed using $\mathrm{R}$ statistical language ( $\mathrm{R}$ Core Team 2015). We used the following packages for $\mathrm{R}$ in the analysis "GenomicRanges" (version 1.16.4) (Lawrence et al. 2013), "TxDb.Hsapiens.UCSC.hg19.knownGene" (version 3.1.2) (http://bioconductor.riken.jp/packages/3.1/data/annotation/html/ TxDb.Hsapiens.UCSC.hg19.knownGene.html), "survival" (version 2.37-7) (http://crantastic.org/packages/survival/versions/32269) and "reshape2" (version 1.4.1) (Wickham 2007). Custom R scripts for determining differentially methylated regions and for predicting and ranking eDMR-gene pairs are provided, together with sample data sets derived from the TCGA, available in the Supplemental Data (see "INFO_README.txt" file in Supplemental Scripts).

\section{Primary human osteoblasts and melanoma cells coculture}

Primary human osteoblasts were isolated using a protocol described by Dillon et al. (2012). In short, trabecular bone was obtained from healthy donors undergoing total knee arthroplasty. Written and informed consent was obtained from all subjects. The protocol was approved by the institutional Ethics Committee at Tel-Aviv Sourasky Medical Center, in accordance with the Helsinki Declaration on the use of human subjects in research. The trabecular bone fragments were diced into small pieces and washed with sterile PBS. The diced bone extracts were then placed on a tissue culture plate with Dulbecco's Modified Eagle Medium (DMEM) supplemented with $20 \%$ fetal bovine serum, $100 \mathrm{U} / \mathrm{mL}$ penicillin, and $100 \mu \mathrm{g} / \mathrm{mL}$ streptomycin (all from Gibco, Life Technologies) and incubated at $37^{\circ} \mathrm{C}$ and $5 \% \mathrm{CO}_{2}$. Five days later, the medium was replaced; medium was then replaced twice a week until the cells reached confluency. Osteoblasts were then seeded $24 \mathrm{~h}$ prior to addition of melanoma cells in a ratio of 1:5 melanoma cells to osteoblasts. In control plates, only melanoma cells were seeded.

\section{Cell culture and FACS sorting}

WM3682 melanoma cells were generously provided by Dr. Levi A. Garraway (Department of Medical Oncology and Center for Cancer Genome Discovery, Dana-Farber Cancer Institute, Boston, MA). Cells were cultured in DMEM medium supplemented with $10 \%$ fetal bovine serum (Sigma-Aldrich) and 1\% penicillin/streptomycin/glutamine (Invitrogen). For establishment of 
stable cell lines, cells were transfected with GFP-expressing plasmid and selected with $1 \mu \mathrm{g} / \mathrm{mL}$ puromicyn (Sigma-Aldrich). For coculture, WM3682-GFP cells were cultured with bone cells for 5 d. Cells were collected by flow cytometry using BD FACSAria cell sorter.

\section{RNA purification and qRT-PCR}

Total RNA was purified from sorted melanoma cells using TRIzol (Invitrogen) according to the manufacturer's instructions, followed by treatment with RNase-free DNase (Qiagen). RNA was quantified based on $\mathrm{OD}_{260 / 280}$. For qRT-PCR analysis, RNA was subjected to one-step qRT-PCR using a MultiScribe RT-PCR kit (Applied Biosystems) and FastStart Universal SYBR Green Master Mix (Roche).

\section{Plasmids and cloning}

EDMRs of the human KIT, KIF14, and CYTL1 loci (Chr4: 55708294-55709294, Chr1:201198480-201199526, Chr4:50196455020678 , respectively) were amplified from human genomic DNA (see Supplemental Table S5 for primer sequences used in cloning). The CYTL1 eDMR (1033 bp) and KIF14 eDMR (1046 bp) fragments were digested with NheI-XhoI restriction enzymes and inserted into the pGL3-promoter vector (Promega) upstream of a minimal promoter and firefly luciferase reporter gene (kindly provided by Professor Eran Bacharach, Department of Cell Research and Immunology, Faculty of Life Sciences, Tel-Aviv University). The KIT eDMR (1000 bp) fragment was cloned into PGL3-promoter reporter plasmid digested with SmaI-XhoI restriction enzymes. The pCDNA3-KIT expression vector was kindly obtained from Prof. Lars Rönnstrand (Division of Translational Cancer Research and Lund Stem Cell Center, Lund University).

\section{In vitro methylation, transfections, and dual luciferase assay}

The KIT, CYTL1, and KIF14 eDMR firefly luciferase reporter vectors were in vitro methylated using the methylase SssI (New England Biolabs), according to the manufacturer's recommendations, followed by purification using the Wizard SV PCR clean-up system (Promega). Successful methylation was verified by restriction enzyme digestion with the methylation-sensitive (HpaII) and methylation-insensitive (MspI) enzymes (New England Biolabs). The digestion patterns were analyzed by agarose gel electrophoresis. WM3682 melanoma cell lines were cotransfected using jetPEI, with a methylated eDMR-luciferase reporter plasmid, unmethylated reporter, or reporter plasmid without eDMR (as control) and with the pRL-plasmid (Promega). Luciferase activity was measured $48 \mathrm{~h}$ after transfection using a Dual Luciferase kit (Promega). Firefly luciferase activity was normalized to the Renilla luciferase.

\section{Invasion assay}

WM3682 melanoma cell lines were transfected with the KIT expression vector or empty vector (as control) using jetPEI. Fortyeight hours post-transfection, an invasion assay was performed as previously described (Golan et al. 2015).

\section{5-aza-deoxycytidine treatment}

WM3682 melanoma cell lines were treated with $10 \mu \mathrm{M}$ 5-aza-dC (Sigma-Aldrich) for $48 \mathrm{~h}$, following by RNA purification and qRT-PCR as described. Baseline expression was established by mock treatment of cells with DMSO.

\section{Acknowledgments}

The authors thank Dr. Levi Garraway for supplying melanoma cultures. C.L. thanks the following for grant support: I-CORE Gene Regulation in Complex Human Disease, Center No. 41/11, Mr. David Brown through the Israel Cancer Association (ICA), Israel Cancer Research Fund (ICRF), Fritz Thyssen Stiftung, Marie Curie Career Integration Grants (CIG), and the Dalya Gridinger Fund. R.E.B. thanks the Charles Clore Foundation.

\section{References}

Andersson R, Gebhard C, Miguel-Escalada I, Hoof I, Bornholdt J, Boyd M, Chen Y, Zhao X, Schmidl C, Suzuki T. 2014. An atlas of active enhancers across human cell types and tissues. Nature 507: 455-461.

Aran D, Hellman A. 2013. DNA methylation of transcriptional enhancers and cancer predisposition. Cell 154: 11 .

Aran D, Hellman A. 2014. Unmasking risk loci: DNA methylation illuminates the biology of cancer predisposition. Bioessays 36: 184-190.

Aran D, Sabato S, Hellman A. 2013. DNA methylation of distal regulatory sites characterizes dysregulation of cancer genes. Genome Biol 14: $\mathrm{R} 21$

Benjamini Y, Hochberg Y. 1995. Controlling the false discovery rate: a practical and powerful approach to multiple testing. J R Stat Soc Ser B 57: 289-300.

Bergman Y, Cedar H. 2013. DNA methylation dynamics in health and disease. Nat Struct Mol Biol 20: 274-281.

Brabletz T. 2012. To differentiate or not-routes towards metastasis. Nat Rev Cancer 12: 425-436.

Braeuer RR, Watson IR, Wu CJ, Mobley AK, Kamiya T, Shoshan E, Bar-Eli M. 2014. Why is melanoma so metastatic? Pigment Cell Melanoma Res 27: 19-36.

Brocks D, Assenov Y, Minner S, Bogatyrova O, Simon R, Koop C, Oakes C, Zucknick M, Lipka Daniel B, Weischenfeldt J, et al. 2014. Intratumor DNA methylation heterogeneity reflects clonal evolution in aggressive prostate cancer. Cell Rep 8: 798-806.

Buecker C, Wysocka J. 2012. Enhancers as information integration hubs in development: lessons from genomics. Trends Genet 28: 276-284.

Bulger M, Groudine M. 2011. Functional and mechanistic diversity of distal transcription enhancers. Cell 144: 327-339.

Corson TW, Huang A, Tsao M-S, Gallie BL. 2005. KIF14 is a candidate oncogene in the $1 \mathrm{q}$ minimal region of genomic gain in multiple cancers. Oncogene 24: 4741-4753.

Craene BD, Berx G. 2013. Regulatory networks defining EMT during cancer initiation and progression. Nat Rev Cancer 13: 97-110.

Dedeurwaerder S, Defrance M, Calonne E, Denis H, Sotiriou C, Fuks F. 2011. Evaluation of the Infinium Methylation 450K technology. Epigenomics 3: 771-784.

Dillon JP, Waring-Green VJ, Taylor AM, Wilson PJ, Birch M, Gartland A Gallagher JA. 2012. Primary human osteoblast cultures. In Bone research protocols, 2nd ed. (ed. Helfrich MH, Ralston SH), pp. 3-18. Humana Press-Springer, New York.

Easwaran H, Tsai H-C, Baylin Stephen B. 2014. Cancer epigenetics: tumor heterogeneity, plasticity of stem-like states, and drug resistance. Mol Cell 54: 716-727.

Ernst J, Kellis M. 2012. ChromHMM: automating chromatin-state discovery and characterization. Nat Methods 9: 215-216.

Esteller M. 2007. Epigenetic gene silencing in cancer: the DNA hypermethylome. Hum Mol Genet 16: R50-R59.

Franchini D-M, Schmitz K-M, Petersen-Mahrt SK. 2012. 5-Methylcytosine DNA demethylation: more than losing a methyl group. Annu Rev Genet 46: 419-441.

Friedl P, Alexander S. 2011. Cancer invasion and the microenvironment: plasticity and reciprocity. Cell 147: 992-1009.

Goding CR, Pei D, Lu X. 2014. Cancer: pathological nuclear reprogramming? Nat Rev Cancer 14: 568-573.

Göke J, Jung M, Behrens S, Chavez L, O'Keeffe S, Timmermann B, Lehrach H, Adjaye J, Vingron M. 2011. Combinatorial binding in human and mouse embryonic stem cells identifies conserved enhancers active in early embryonic development. PLoS Comput Biol 7: e1002304.

Golan T, Messer AR, Amitai-Lange A, Melamed ZE, Ohana R, Bell RE, Kapitansky O, Lerman G, Greenberger S, Khaled M. 2015. Interactions of melanoma cells with distal keratinocytes trigger metastasis via Notch signaling inhibition of MITF. Mol Cell 59: 664-676.

He B, Chen C, Teng L, Tan K. 2014. Global view of enhancer-promoter interactome in human cells. Proc Natl Acad Sci 111: E2191-E2199.

Heintzman ND, Hon GC, Hawkins RD, Kheradpour P, Stark A, Harp LF, Ye Z, Lee LK, Stuart RK, Ching CW, et al. 2009. Histone modifications at 
human enhancers reflect global cell-type-specific gene expression. Nature 459: 108-112.

Hnisz D, Abraham BJ, Lee TI, Lau A, Saint-André V, Sigova AA, Hoke HA, Young RA. 2013. Super-enhancers in the control of cell identity and disease. Cell 155: 934-947.

Holst F, Moelans CB, Filipits M, Singer CF, Simon R, van Diest PJ. 2012. On the evidence for ESR1 amplification in breast cancer. Nat Rev Cancer 12: 149.

Hon GC, Rajagopal N, Shen Y, McCleary DF, Yue F, Dang MD, Ren B. 2013. Epigenetic memory at embryonic enhancers identified in DNA methylation maps from adult mouse tissues. Nat Genet 45: 1198-1206.

Jones PA. 2012. Functions of DNA methylation: islands, start sites, gene bodies and beyond. Nat Rev Genet 13: 484-492.

Korpal M, Lee ES, Hu G, Kang Y. 2008. The miR-200 family inhibits epithelial-mesenchymal transition and cancer cell migration by direct targeting of E-cadherin transcriptional repressors ZEB1 and ZEB2.J Biol Chem 283: 14910-14914.

Kwon YJ, Lee SJ, Koh JS, Kim SH, Lee HW, Kang MC, Bae JB, Kim YJ, Park JH. 2012. Genome-wide analysis of DNA methylation and the gene expression change in lung cancer. J Thorac Oncol 7: 20-33.

Lam MT, Li W, Rosenfeld MG, Glass CK. 2014. Enhancer RNAs and regulated transcriptional programs. Trends Biochem Sci 39: 170-182.

Lawrence $\mathrm{M}$, Huber W, Pagès $\mathrm{H}$, Aboyoun P, Carlson M, Gentleman R, Morgan MT, Carey VJ. 2013. Software for computing and annotating genomic ranges. PLoS Comput Biol 9: e1003118.

Lee WJ, Roberts-Thomson SJ, Holman NA, May FJ, Lehrbach GM, Monteith GR. 2002. Expression of plasma membrane calcium pump isoform mRNAs in breast cancer cell lines. Cell Signal 14: 1015-1022.

Ma L, Young J, Prabhala H, Pan E, Mestdagh P, Muth D, Teruya-Feldstein J, Reinhardt F, Onder TT, Valastyan S. 2010. miR-9, a MYC/MYCN-activated microRNA, regulates E-cadherin and cancer metastasis. Nat Cell Biol 12: $247-256$

Marzese DM, Scolyer RA, Huynh JL, Huang SK, Hirose H, Chong KK, Kiyohara E, Wang J, Kawas NP, Donovan NC. 2014. Epigenome-wide DNA methylation landscape of melanoma progression to brain metastasis reveals aberrations on homeobox D cluster associated with prognosis. Hum Mol Genet 23: 226-238.

Meacham CE, Morrison SJ. 2013. Tumour heterogeneity and cancer cell plasticity. Nature 501: 328-337.

Meyers M, Balch C. 1998. Diagnosis and treatment of metastatic melanoma. In Cutaneous melanoma, 3rd ed. (ed. Balch CM, et al.). Quality Medical Publishing, St. Louis, MO.

Mitra A, Fillmore RA, Metge BJ, Rajesh M, Xi Y, King J, Ju J, Pannell L, Shevde LA, Samant RS. 2008. Large isoform of MRJ (DNAJB6) reduces malignant activity of breast cancer. Breast Cancer Res 10: R22.

Murata Y, Minami Y, Iwakawa R, Yokota J, Usui S, Tsuta K, Shiraishi K, Sakashita S, Satomi K, Iijima T. 2014. ECT2 amplification and overexpression as a new prognostic biomarker for early-stage lung adenocarcinoma. Cancer Sci 105: 490-497.

Pletscher-Frankild S, Pallejà A, Tsafou K, Binder JX, Jensen LJ. 2015. DISEASES: text mining and data integration of disease-gene associations. Methods 74: 83-89.
Pogribny IP, Beland FA. 2009. DNA hypomethylation in the origin and pathogenesis of human diseases. Cell Mol Life Sci 66: 2249-2261.

R Core Team. 2015. R: a language and environment for statistical computing. R Foundation for Statistical Computing, Vienna, Austria. http://www.Rproject.org/.

Richardson B. 2003. Impact of aging on DNA methylation. Ageing Res Rev 2: $245-261$.

Rosenbloom KR, Sloan CA, Malladi VS, Dreszer TR, Learned K, Kirkup VM, Wong MC, Maddren M, Fang R, Heitner SG, et al. 2013. ENCODE data in the UCSC Genome Browser: year 5 update. Nucleic Acids Res 41(D1): D56-D63.

Sharif TR, Sharif M. 1999. Overexpression of protein kinase C $\varepsilon$ in astroglial brain tumor derived cell lines and primary tumor samples. Int JOncol 15: 237-243.

Stone A, Zotenko E, Locke WJ, Korbie D, Millar EKA, Pidsley R, Stirzaker C, Graham P, Trau M, Musgrove EA, et al. 2015. DNA methylation of oestrogen-regulated enhancers defines endocrine sensitivity in breast cancer. Nat Commun 6: 7758

Taberlay PC, Statham AL, Kelly TK, Clark SJ, Jones PA. 2014. Reconfiguration of nucleosome-depleted regions at distal regulatory elements accompanies DNA methylation of enhancers and insulators in cancer. Genome Res 24: 1421-1432.

Tam WL, Weinberg RA. 2013. The epigenetics of epithelial-mesenchymal plasticity in cancer. Nat Med 19: 1438-1449.

Teng L, He B, Wang J, Tan K. 2015. 4DGenome: a comprehensive database of chromatin interactions. Bioinformatics 31: 2560-2564.

Tian Q, Frierson HF, Krystal GW, Moskaluk CA. 1999. Activating c-kit gene mutations in human germ cell tumors. Am J Pathol 154: 1643-1647.

Verras M, Brown J, Li X, Nusse R, Sun Z. 2004. Wnt3a growth factor induces androgen receptor-mediated transcription and enhances cell growth in human prostate cancer cells. Cancer Res 64: 8860-8866.

Wen M, Wang H, Zhang X, Long J, Lv Z, Kong Q, An Y. 2012. Cytokine-like 1 is involved in the growth and metastasis of neuroblastoma cells. Int $J$ Oncol 41: $1419-1424$.

Whyte WA, Orlando DA, Hnisz D, Abraham BJ, Lin CY, Kagey MH, Rahl PB, Lee TI, Young RA. 2013. Master transcription factors and mediator establish super-enhancers at key cell identity genes. Cell 153: 307-319.

Wickham H. 2007. Reshaping data with the reshape package. J Stat Softw 21: $1-20$.

You JS, Jones PA. 2012. Cancer genetics and epigenetics: two sides of the same coin? Cancer Cell 22: 9-20.

Zhou VW, Goren A, Bernstein BE. 2011. Charting histone modifications and the functional organization of mammalian genomes. Nat Rev Genet 12: 7-18.

Zhu X-L, Liang L, Ding Y-O. 2008. Overexpression of FMNL2 is closely related to metastasis of colorectal cancer. Int J Colorectal Dis 23: 1041-1047.

Ziller MJ, Gu H, Müller F, Donaghey J, Tsai LT-Y, Kohlbacher O, De Jager PL, Rosen ED, Bennett DA, Bernstein BE. 2013. Charting a dynamic DNA methylation landscape of the human genome. Nature 500: 477-481.

Received July 21, 2015; accepted in revised form February 19, 2016. 


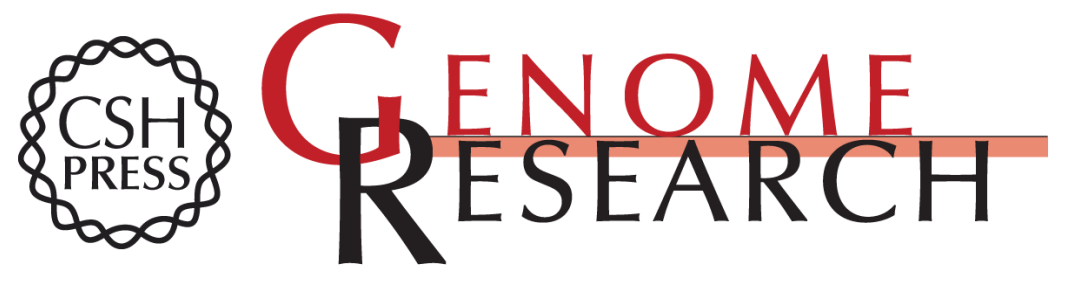

\section{Enhancer methylation dynamics contribute to cancer plasticity and patient mortality}

Rachel E. Bell, Tamar Golan, Danna Sheinboim, et al.

Genome Res. 2016 26: 601-611 originally published online February 23, 2016

Access the most recent version at doi:10.1101/gr.197194.115

\section{Supplemental} Material

References

Creative

Commons

License

Email Alerting Service
http://genome.cshlp.org/content/suppl/2016/04/06/gr.197194.115.DC1

This article cites 56 articles, 4 of which can be accessed free at: http://genome.cshlp.org/content/26/5/601.full.html\#ref-list-1

This article is distributed exclusively by Cold Spring Harbor Laboratory Press for the first six months after the full-issue publication date (see

$\mathrm{http}: / / g$ enome.cshlp.org/site/misc/terms.xhtml). After six months, it is available under a Creative Commons License (Attribution-NonCommercial 4.0 International), as described at http://creativecommons.org/licenses/by-nc/4.0/.

Receive free email alerts when new articles cite this article - sign up in the box at the top right corner of the article or click here.

\section{Affordable, Accurate Sequencing.}

To subscribe to Genome Research go to:

https://genome.cshlp.org/subscriptions 